\title{
Performance characteristics of InGaAs/GaAs and GaAs/InGaAlAs coherently strained superiattice photodiodes
}

\author{
Utpai Das, Yousef Zebda, Pallab Bhattacharya, and Albert Chin \\ Solid State Electronics Laboratory and Center for High Frequency Microelectronics, Department of \\ Electrical Engineering and Computer Science, University of Michigan, Ann Arbor, Michigan 48109-2122
}

(Received 26 June 1987; accepted for publication 13 August 1987)

The properties of $\mathrm{In}_{0.24} \mathrm{Ga}_{0.76} \mathrm{As} / \mathrm{GaAs}$ and GaAs/ $/ \mathrm{In}_{0.05} \mathrm{Ga}_{0.58} \mathrm{Al} \mathrm{I}_{0.37}$ As superlattice photodiodes grown by molecular beam epiaxy have been investigated. From the temporal response characteristics, deconvolved rise times $\sim 60-100 \mathrm{ps}$ are obtained. The measured responsivities of the photodiodes with dark currents of $5-10 \mathrm{nA}$ at $10 \mathrm{~V}$ are $\sim 0.4 \mathrm{~A} / \mathrm{W}$, which correspond to peak external quantum efficiencies of $\sim 60 \%$. These resuits indicate that very high performance photodiodes can be realized with strained layers.

Superiattice (SL) and multiquantum well (MQW) photodiodes are of interest for a variety of reasons. First, they allow a talloring of the spectrai response characieristics by a variation of the quantum well parameters. Second, the initial proposal ${ }^{1.2}$ of erhancement of impact ionization coefficient ratios, $\alpha / \beta$, in these artificial materials for low-roise applications has been borne out by measurements. ${ }^{3-5}$ Third, a certain amount of tunability is provided by the shife in the pesition of the exciton resonances with applied electric field perpendicular to the layers. In this letter we demonstrate that very high-speed mulliquantum well photodiodes can be realized with strained-layer superlattices (SLS's). In particular, we have characterized devices made with $\mathrm{In}_{0.24} \mathrm{Ga}_{0.76} \mathrm{As} / \mathrm{GaAs}$ and $\mathrm{GaAs} / \mathrm{In}_{0.05} \mathrm{Ga}_{0.58} \mathrm{Al}_{0.37} \mathrm{As}$ SLS's. The devices aiso exhibit external quantum efficiencies of $\sim 60 \%$.

The MQW $p-i-n$ photodiode structures were grown by molecular beam epitaxy on conducting Si-doped GaAs substrates. The schematics of the structures with the two strained MOW systems are shown in Figs. 1 (a) and $1(b)$. The InGaAs/GaAs SLS was grown at $520^{\circ} \mathrm{C}$, while the GaAs/IrGaAlAs structure was grown at $600^{\circ} \mathrm{C}$. The SLS absorption regions in the two structures are 1.2 and $1.0 \mu \mathrm{m}$ thick, respectively. The mismatch values in the InGaAs/ GaAs and GaAs/InGaAiAs SLS systems investigated by us are 1.4 and $0.35 \%$, respectively. Low-temperature absorption measurements were made to ascertain the structural quality of the SLS regions. The measurements were made with a tungsten-halogen source, a $1-m$ Jarell- $\mathbf{A}$ sh scanning spectrometer, and a liquid $\mathbb{N}_{2}$-cooled photomultiplier. The spectra were recorded after suitable amplification by lock-in techniques. The spectra are shown in Figs. 2(a) and 2(b). In the spectrum of the GaAs/AlGalnAs MOW, the two dominant transitions result from the HHI $(n=1$ electron to heavy hole) and LH1 ( $n=1$ electron to light hole) transitions. These are, as expected, separated by $6-7 \mathrm{meV}$. The spectrum for the InGaAs/GaAs MQW shows one dominant peak, which we believe is the HHI transition and several very weak ones at higher energies. The one closest to the main peak, and separated from it by $17 \mathrm{meV}$, is believed to be due to a transition from $n=1$ electron to $n=2$ heavy-hole subband. This transition is, in general, forbicden, but be- comes possible due to band mixing, and hence the intensity is very small.

The device fabrication procedure is as foliows. A square mesa $\left(20 \times 25 \mathrm{~km}^{2}\right)$ defining the active area is formed by etching with $1 \mathrm{H}_{3} \mathrm{PO}_{4}: 1 \mathrm{H}_{2} \mathrm{O}_{2}: 8 \mathrm{H}_{2} \mathrm{O}$ up to the $n+$ InGaAs or AlGaAs layer. $3000 \mathrm{~A} \mathrm{SiO}_{2}$ is deposited on the whole structure and openings of dimension $5 \times 5 \mu \mathrm{m}^{2}$ are made by etching with bufiered $\mathrm{HF}$ for the p-type contacts. $500 / 3500 \AA$ Ti/Au and 300/400/3500 $\AA \mathrm{Ni} / \mathrm{Ge} / \mathrm{Au} p$ - and n-type contacts are formed on the top and back, respectively. The calculated zero-bias junction capacitance of the diodes is less than $0.1 \mathrm{pF}$. Excellent rectifying characteristics were obtained. The dark current at $10 \mathrm{~V}$ is $5-10 \mathrm{nA}$ for both material systems.

The spectral response characteristics of the devices were

\begin{tabular}{|c|c|c|}
\hline$p^{+}$ & GaAs & $50 \AA$ \\
\hline$p^{r}$ & $\mathrm{Al}_{0.1} \mathrm{Ga}_{0.9} \mathrm{As}$ & $200 \AA$ \\
\hline Undoped & $\ln _{0.24} \mathrm{Gat}_{0.78} \mathrm{As}$ & GaAs SLS \\
\hline$L_{z}=100 \AA$ & - $L_{B}=150 \AA$ & $1.2 \mu \mathrm{m}$ \\
\hline$n^{+}$ & $\ln _{0.1} \mathrm{Ga}_{0.9} \mathrm{As}$ & $0.4 \mathrm{~km}$ \\
\hline $\mathrm{n}^{+}$ & GaAs & $0.3 \mu \mathrm{m}$ \\
\hline
\end{tabular}

(a)

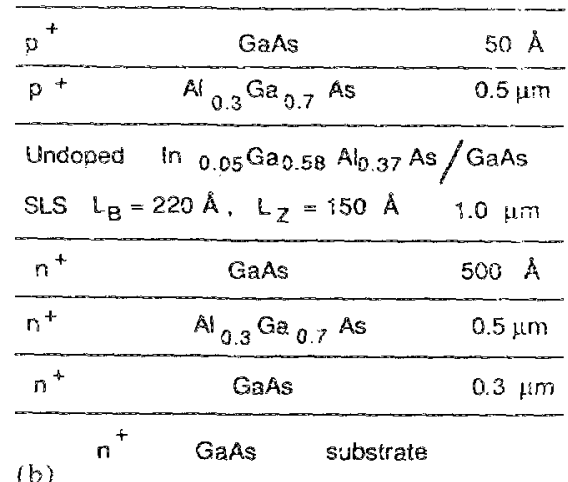

FIG. 1. Schemaitics of (a) $\operatorname{In}_{0.24} \mathrm{Ga}_{0.76} \mathrm{As} / \mathrm{GaAs}$ SLS and (b) GaAsf $\mathrm{In}_{0.05} \mathrm{Ga}_{0.58} \mathrm{Al} \mathrm{l}_{0.37} \mathrm{As}$ SLS photoliodes grown by molecular beam epitaxy. 



FIG. 2. Measured low-lemperarure absorption spectra of (a) IncraAs/

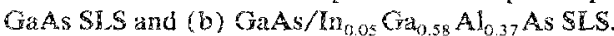

recorded with a monochromatic light source and lock-in amplification of the detected photocurrent. The measured photocurrents were calibrated with a Si photodiode. The spectral response of the diodes at a reverse bias of $15 \mathrm{~V}$ is shown in Figs. 3(a) and 3(b). The maximum responsivity of $\sim 0.4$ $A / W$ is measured in both tnaterial systems, indicating that this value is almost intrinsic to the device design. The maximum external quantum efficiency for both types of SLS devices is $-60 \%$ at a bias of $-15 \mathrm{~V}$ and $0.75 \mu \mathrm{m}$ wavelength light.

For the high-speed frequency response measurements, the photodiodes were mounted on a $50-\Omega$ microstripline package de bias to the diode was provided through a RP $11612 \mathrm{~A}$ bias-Tee with an operating range of $45 \mathrm{MHz}$ to 26.5 Griz. Optical excitation was provided with $100 \mathrm{ps}$ pulses from a Northcoast 810 AlGaAs ( $850 \mathrm{~nm}$ ) laser diode with the output focused to a $50-\mu \mathrm{m}$ spot on the device. The response of the photodiode to the optical pulses was observed on a sampling scope with an $\$-4$ sampling head having a rise time of 25 ps. The high-frequency coaxial cables used in the circuitry have an approximate rise time of $10 \mathrm{ps}$. Figures $4(a)$ and $4(b)$ show the temporal response of the photodiodes as observed on the sampling scope. From these data the deconvolved rise times are calculated to be 100 and $60 \mathrm{ps}$ in the InGaAs/GaAs and GaAs/InGaAlAs photodiodes, respectively. The corresponding full widths at half-maxi-
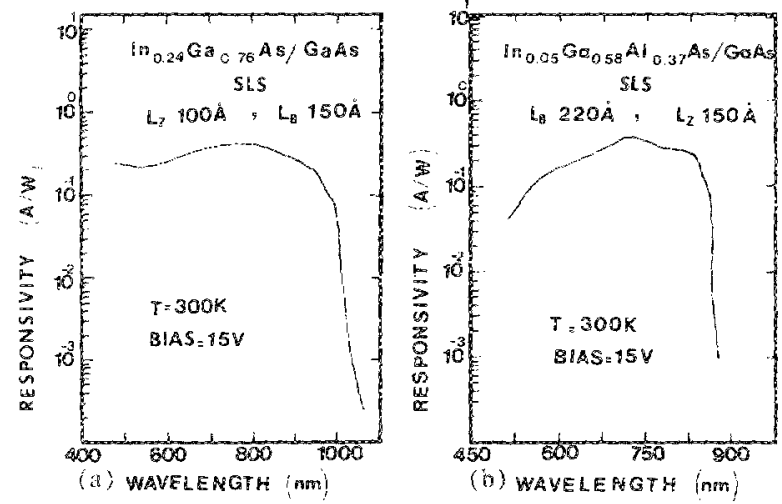

FIG. 3. Measured spectral response th room temperature of (a) InGaAs/ GaAs and (b) GaAs/IrAlGaAs $S L$, photodiode under a reverse bias of 15 $V$. At this bias the $i$ region is fully depleted in both cases.
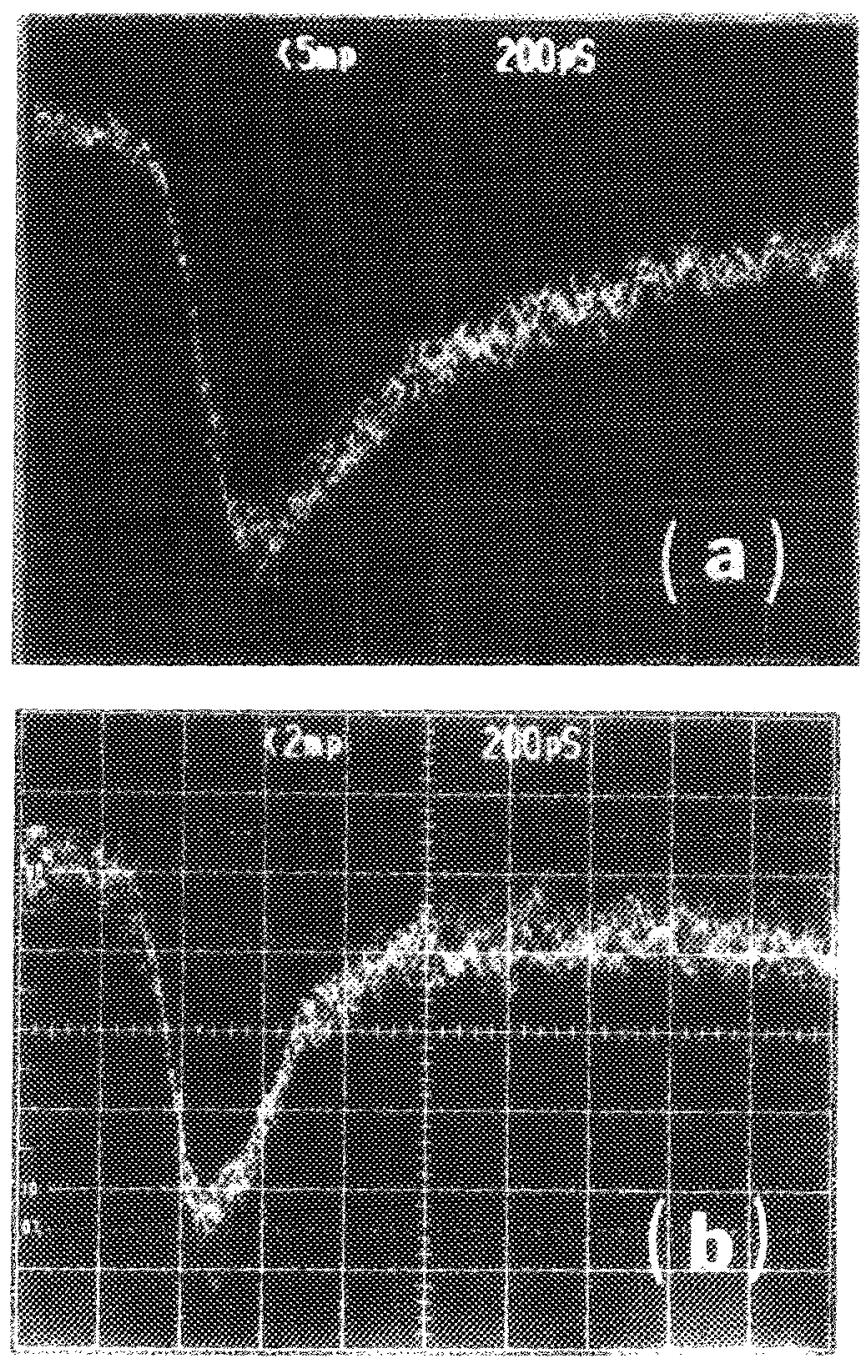

PIG. 4. Impulse response of (a) InGaAs/GaAs and (b) GaAs/InGaAJAs photodiodes to a 850 -nm AlGaAs laser with a pulse width (FWMM) of 100 ps.

mum (FWMM) of the responses are 670 and $260 \mathrm{ps}$, respectiveiy.

It is to be realized that the intrinsic speed of these devices is ultimately limited by the $R C$ time constant associated with the $p^{+}$contact pad $\left(75 \times 75 \mu \mathrm{m}^{2}\right)$ separated by 3000 $\AA$ of dielectric from the $n+$ layer below them. The caiculated capacitance is $\sim 700 \mathrm{FP}$, which is an order higher than the diode junction capacitance. In fact, InGaAs $p$-i-n diodes grown on semi-insulating InP substrates having a $0.75-\mu m$ absorption layer and having an identical geometry exhibit temporal responses with a rise time of 22 ps and FWHM of $27 \mathrm{ps}$. The $R C$ time constant in the SLS devices, taking into account the pad capacitance, is approximately $36 \mathrm{ps}$. It is clear that a monolithic structure fabricated on semi-insulating substrates is more desirable for high-speed devices.

The rise times of $60 \mathrm{ps}$ in the impulse response of the $\mathrm{GaAs} / \mathrm{InGaAlAs}$ SLS devices reflect the carrier transport mechanism through the superiatice region under the applied bias, since the incident putsed photoexcitation has an energy a little higher than the GaAs well band gap. As pointed out by larsson et al., ${ }^{6}$ these values of the response time indicate carrier transit by feid-erhanced emission and some 
tunneling through barriers. In the case of the InGaAs/GaAs SLS, the pulsed photoexcitation is observed above the barrier energy and the photoexcited carriers are transported almost as in bulk materials. Long tails in the temporal response, as observed in our diodes, have been previously reported by other authors. ${ }^{6,7}$ We believe these long fall times result from a certain amount of carrier trapping in the wells, as shown by arker et $a l^{8}$ and also from carrier trapping in bulk and interface traps and defects in the SLS. The fact that the response of the GaAs/InGaAIAs SLS with a $0.35 \%$ mismatch is much faster than the InGaAs/GaAs SLS with a $1.4 \%$ mismatch indicates that the higher strain and possibly higher interface defects in the latter might be responsible.

It is clear from the data presented above that fast photodiodes can be fabricated with strained-layer superlattices and their performance is comparable to bulk and iatticematched SL photodiodes. We have demonstrated good performance in two types of SLS photodiodes in which the wells of the SL region are under tensile or compressive strain. More optimum device design can certainly lead to the use of these devices for data transmission at the rate of several gigabits per second. In addition, we have recently shown ${ }^{9}$ that a higher responsivity can be obtained in the case of the GaAs/InGaAlAs SLS near the exciton resonances due to a merger of the light- and heavy-hole bands for certain alloy compositions. In the sample studied here, with $\sim 5 \% \mathrm{In}$, two resonances are still separate, as seen in Fig. 2, and therefore, the quantum efficiencies in the two strained samples are comparable. With an increase in the absorption coefficient, it should be possible to use thinner absorption regions, thereby reducing the transit time of photoexcited carriers across it.

The authors wish to thank Dr. G. P. Kothiyal for performing the low-temperature absorption measurements and Professor I. Singh for stimulating discussions. The work was supported by the National Aeronauticai and Space Agency under grant NAG $1-555$ and the Amy Research Office under contract DAAL03-87-K-0007.

${ }^{\prime}$ R. Chin, N. Holonyak, Jr, G. E. Stillman, J.Y. Tang, and K. Hess, Eleetron. Lett. $\$ 6,467$ (1980).

${ }^{2}$ T. Capasso, W. T. Tsang, A. L. Futchinson, and G. F. Williams, Appl Phys. Lett. 40, 38 (1982).

3 -Y.Juang, U. Das, Y. Nashimoto, and P. Bhattacharya, Appl. Phys. Lett. $47,972(1985)$

HF. Capasso, W. T. Tsang, and G. F. Whlliams, MEEE Trans. Electron Devices ED-30, 381 (1983).

"F. Osaku and T. Mikawa, IEEE J. Quantum Electron. QE-22, 471 (1986).

A. Larsson, A. Yariv, R. Tell, J. Maserjian, and S. T. Eng, Appl. Phys. Lett. 47,866 (1985).

'F. Beltram, J. Allam, F. Capasso, U. Koren, and B. Miller, Appl. Phys. Lett. 50, 1170 (1987).

${ }^{8}$ D. G. Parker, N. R. Couch, M. J. Kelly, and T. M. Kerr, Appl. Phys. Leit. 49,939 (1986).

GG. P. Kothiyal, S. Hong, N. Debbar, P. Bhattacharya, and I. Singh, Appl. Phys. Lets. 51, 1091 (1987). 